\title{
Maximizing Capacity with Power Control under Physical Interference Model in Simplex Mode*
}

\author{
Peng-Jun Wan, Chao Ma, Shaojie Tang, and Boliu Xu \\ Illinois Institute of Technology, Chicago, IL 60616
}

\begin{abstract}
This paper addresses the join selection and power assignment of a largest set of given links which can communicate successfully at the same time under the physical interference model in the simplex mode. For the special setting in which all nodes have unlimited maximum transmission power, Kesselheim [8] developed an constant approximation algorithm. For the general setting in which all nodes have bounded maximum transmission power, the existence of constant approximation algorithm remains open. In this paper, we resolve this open problem by developing a constant-approximation algorithm for the general setting in which all nodes have bounded maximum transmission power.
\end{abstract}

\section{Introduction}

A wireless network is specified, in its most general format, by a triple $(V, A, \mathcal{I})$, where $V$ is the set of networking nodes, $A$ is the set of communication links among $V$, and $\mathcal{I}$ is the collection of sets of links in $A$ which can transmit successfully at the same time. Each set in $\mathcal{I}$ is referred to as an independent set. The maximum size of the independent sets is called the independence number of the network, and is denoted by $\alpha$. The problem of finding a maximum independent subset of a give set $B \subseteq A$ is referred to as Maximum Independent Set of Links (MISL).

This paper studies MISL in wireless networks under the physical interference model in the simplex mode. We assume that all nodes in $V$ lie in an Euclidean plane. The distance between any pair of nodes $u$ and $v$ is denoted by $\ell(u, v)$. The signal strength attenuates with a path loss factor $\eta r^{-\kappa}$, where $r$ is the distance from the transmitter, $\kappa$ is path-loss exponent, and $\eta$ is the reference loss factor. The signal quality perceived by a receiver is measured by the signal to interference and noise ratio (SINR), which is the quotient between the power of the wanted signal and the total power of unwanted signals and the ambient noise $\xi$. In order to correctly interpret the wanted signal, the SINR must exceed certain threshold $\sigma$. Thus, for any pair of nodes $a=\{u, v\}$ to commutate with each other even without any interference, their transmission power should exceed

$$
p_{0}(a)=\frac{\sigma \xi}{\eta} \ell(a)^{\kappa},
$$

\footnotetext{
* This work was supported in part by NSF of USA under grants CNS-0831831 and CNS-0916666.
} 
where $\ell(a)$ is the length of $a$. Suppose that all nodes have maximum transmission power $P$. Then, the largest possible set of communication links, denoted by $A$, consists of all possible pairs $a$ of nodes satisfying that $p_{0}(a)<P$. For a specific power assignment $p$, the set of communication links, denoted by $A_{p}$, consists of all possible pairs $a$ of nodes satisfying that

$$
p_{0}(a)<p(a) \leq P
$$

Clearly, $A_{p} \subseteq A$. In the simplex mode of the physical interference model, the communications between a pair of nodes are unidirectional. For a specific power assignment $p$ and any pair of distinct links $a$ and $b$ in $A_{p}$, when they transmit at the same time the interference of $a$ toward $b$ is $p(a) \cdot \eta \ell(a, b)^{-\kappa}$, where $\ell(a, b)$ is the distance between the sender of $a$ and the receiver of $b$. Let $\mathcal{I}_{p}$ denote the collection of all subsets of $A_{p}$ which can communicate successfully at the same time with the power assignment $p$.

There are two variants of the MISL under the physical interference model in the simplex mode, with or without power control. In the variant without power control, a power assignment $p$ is pre-specified and the network instance of MISL is simply $\left(V, A_{p}, \mathcal{I}_{p}\right)$. If $p(a)$ is a constant for all $a \in A$, and it is said be uniform. If $p(a)=P$ for all $a \in A, p$ is referred to as the canonical uniform power assignment. A power assignment $p$ is said to be monotone if $p(a)$ is non-decreasing with $\ell(a)$, to be sub-linear if $p(a) / \ell(a)^{\kappa}$ is non-increasing with $\ell(a)$. MISL with uniform power assignment is NP-hard 3]. For MISL with uniform power assignment in the Euclidean plane and $\kappa>2$, Wan et al. [10, developed a constant-approximation algorithm, and Halldórsson and Wattenhofer 7] independently claimed a constant approximation algorithms without proof and the explicit approximation bound. For MISL with any monotone and sub-linear power assignment in arbitrary metric space, Halldórsson and Mitra 6] gave a constant-approximation algorithm. Other weaker (or even false) results on MISL with fixed power assignment have been obtained in [1/34/12].

In the variant with power control, the power assignment is part of the output rather than an input. The network instance is $(V, A, \mathcal{I})$ where $\mathcal{I}$ is the union of $\mathcal{I}_{p}$ for all possible power assignments $p$. For any $B \subseteq A$, MISL with power control seeks a power assignment $p$ and a subset $I \in \mathcal{I}_{p}$ of $B$ such that $|I|$ is maximized. MISL with power control is also NP-hard [1. Approximation algorithms for MISL with power control has been studied in [1] and [8]. In [11], Wan et al. assumed that a bounded set $S$ of possible values of transmission power of all nodes and obtained an $O(\beta)$-approximation algorithm, where $\beta$ is the power diversity of $S$, defined to be smallest integer $k$ such that there exists a partition of $S$ into $k$ subsets in each of which any two elements differ by a factor of at most two. In [8], Kesselheim assumed unlimited maximum transmission power and developed a constant-approximation algorithm in the fading metric and a logarithmic approximation algorithm in the general metric. The assumption on unlimited maximum transmission power is essential in their algorithm as it would technically avoid the major technical obstacle due to the ambient noise. However, such strong assumption is not only impractical, but 
also effectively results in the single-hop wireless network in which every pair of nodes can directly with each other, as opposed to the targeted multihop wireless network. Other weaker results on MISL with power control have been obtained in [2] and [5].

The main contribution of this paper is the design and analysis of a constantapproximation algorithm for MISL with power control in which all nodes have bounded maximum transmission power and $\kappa>2$.

\section{Preliminaries}

We define a constant

$$
\gamma=16 \zeta(\kappa-1)+8 \zeta(\kappa)-6
$$

where $\zeta(x)=\sum_{j=1}^{\infty} j^{-x}$ is the Riemann zeta function. The following geometric lemma can be proved using the same argument as used in Lemma 1 in [10].

Lemma 1. Consider a node $u$ and a set $W$ of nodes other than $u$ in the plane satisfying that $W \cup\{u\}$ have mutual distances at least one. Then,

$$
\sum_{w \in W} \frac{1}{\ell(w, u)^{\kappa}} \leq \gamma
$$

Consider a power assignment $p$. The relative interference (also termed affectance in [6]) of a link $a \in A_{p}$ toward another link $b \in A_{p} \backslash\{a\}$, is defined to be

$$
R I_{p}(a, b):=\sigma \frac{p(a)}{p(b)-p_{0}(b)}\left(\frac{\ell(b)}{\ell(a, b)}\right)^{\kappa} .
$$

It's easy to verify for any subset $I$ of $A_{p}, I \in \mathcal{I}_{p}$ if and only for each $b \in I$,

$$
\sum_{a \in I \backslash\{b\}} R I_{p}(a, b)<1 .
$$

In addition, we have the following theorem.

Theorem 1. Consider a power assignment $p$. For any subset $B \subseteq A_{p}$ whose total relative interference is at most $|B| \phi$ for some $\phi \geq 1$, there is a subset $I$ of $B$ such that $|I| \geq \frac{|B|}{4 \phi}$ and $I \in \mathcal{I}_{p}$.

Due to the space limitation, the proof for the above theorem is omitted.

\section{Power Control and Link Selection}

A link $a \in A$ is called a long link if $p_{0}(a) \geq P / 4$, and a short link otherwise. Fix an ordering $\prec$ of $A$ in the increasing order of length. For each link $a \in A$ and any $S \subseteq A$, we denote by $S_{\prec}(a)$ (respectively, $S_{\succ}(a)$ ) the set of links in $S$ preceding (respectively, succeeding) $a$ in the ordering $\prec$. In this section, 
we will first present a constant-approximation LongIS for MISL with power control restricted to long links in Subsection 3.1. Then, we develop a constantapproximation ShortIS for MISL with power control restricted to short links in Subsection 3.1. Finally, we combine these two algorithm to get a constantapproximation for MISL with power control but without any restriction on link lengths in Subsection 3.3 .

\subsection{Long Links}

Let $\mathcal{A}_{1}$ denote the constant-approximation algorithm for MISL with uniform power assignment, given in [10] (or alternatively, the algorithm given in [6]), and $\mu_{1}$ be its approximation radio. Consider a set $B$ of long links. The algorithm LongIS adopts the canonical uniform power assignment denoted by $q$ and selects an independent set $I$ of $B$ under $q$ using the algorithm $\mathcal{A}_{1}$. The next theorem gives a lower bound on $|I|$.

Theorem 2. $|I| \geq \alpha(B) /\left(16 \mu_{1}\right)$.

Proof. Let $I^{*}$ be a maximum independent set of $B$ and $p$ be its associated power assignment. Then, $\left|I^{*}\right|=\alpha(B)$. Since all links in $B$ are long, for any $a \in B$ we have

$$
P / 4 \leq p_{0}(a)<p(a) \leq P,
$$

Thus, for any pair of links $a$ and $b$ in $I^{*}$, we have

$$
\frac{R I_{q}(a, b)}{R I_{p}(a, b)}=\frac{q(a)}{p(a)} \frac{p(b)-p_{0}(b)}{q(b)-p_{0}(b)}=\frac{P}{p(a)} \frac{p(b)-p_{0}(b)}{P-p_{0}(b)} \leq \frac{p(b)}{p(a)}<4 .
$$

Therefore, the total relative interference of $I^{*}$ under the power assignment $q$ is less than 4. By Theorem 1, there is a subset $I^{\prime}$ of $I^{*}$ such that $\left|I^{\prime}\right| \geq\left|I^{*}\right| / 16$ and $I^{\prime}$ is independent under the power assignment $q$. Hence,

$$
|I| \leq \mu_{1}\left|I^{\prime}\right| \leq 16 \mu_{1}\left|I^{*}\right|=16 \mu_{1} \alpha(B) .
$$

So, the theorem follows.

\subsection{Short Links}

Consider a set $I$ of short links, and let $a_{1}, a_{2}, \cdots, a_{|I|}$ be the sequence of links in $I$ sorted in the ordering $\prec$. Set

$$
p\left(a_{|I|}\right)=2 \frac{\sigma \xi}{\eta} \ell\left(a_{|I|}\right)^{\kappa}
$$

and for each $i$ from $|I|-1$ down to 1 , set

$$
p\left(a_{i}\right)=2 \sigma \ell\left(a_{i}\right)^{\kappa}\left(\sum_{j=i+1}^{|I|} \frac{p\left(a_{j}\right)}{\ell\left(a_{j}, a_{i}\right)^{\kappa}}+\frac{\xi}{\eta}\right) .
$$


This power assignment is referred to as the canonical iterative power assignment to $I$. We shall first provide a sufficient condition on $I$ for the canonical iterative power assignment to $I$ being feasible.

For any two distinct links $a$ and $b$, define

$$
c(a, b)=\left(\frac{\min \{\ell(a), \ell(b)\}}{\ell(a, b)}\right)^{\kappa} .
$$

We define the complete digraph $G$ on $A$ in which the weight of each arc $(a, b)$ is $c(a, b)$. Let

$$
\tau=\left(\sqrt{1+(4 \sigma(1+\sigma))^{-1 / \kappa}}-1\right)^{\kappa} .
$$

It is the unique positive root of the equation

$$
4 \sigma(1+\sigma) \tau\left(2+\tau^{1 / \kappa}\right)^{\kappa}=1
$$

As the result,

$$
2 \sigma \tau=\frac{1}{2(1+\sigma)\left(2+\tau^{1 / \kappa}\right)^{\kappa}} .
$$

Theorem 3. Consider a subset I of short links in A satisfying that for any $a \in I$,

$$
\begin{aligned}
& \sum_{b \in I_{\prec}(a)} c(a, b) \leq \tau, \\
& \sum_{b \in I_{\prec}(a)} c(b, a) \leq \tau, \\
& \sum_{b \in I_{\succ}(a)} c(b, a) \leq \frac{1}{4 \sigma} .
\end{aligned}
$$

Let $p$ be the canonical iterative power assignment to $I$. Then, $p(a) \leq P$ for any $a \in I$ and $I$ is independent under $p$.

Proof. We first claim that $p\left(a_{i}\right) \leq P$ for each $1 \leq 1 \leq|I|$. We prove this claim by downward induction on $i$. For the induction basis,

$$
p\left(a_{|I|}\right)=2 \sigma \ell\left(a_{|I|}\right)^{\kappa} \leq P / 2<P .
$$

For the inductive step, consider $1 \leq 1<|I|$ and assume that $p\left(a_{j}\right) \leq P$ for each $i<j \leq|I|$. Then,

$$
\begin{aligned}
p\left(a_{i}\right) & \leq 2 \sigma\left(\max _{i<j \leq|I|} p\left(a_{j}\right)\right) \sum_{k=j+1}^{|I|}\left(\frac{\ell\left(a_{i}\right)}{\ell\left(a_{j}, a_{i}\right)}\right)^{\kappa}+2 \frac{\sigma \xi}{\eta} \ell\left(a_{i}\right)^{\kappa} \\
& \leq 2 \sigma P \sum_{k=j+1}^{|I|} c\left(a_{j}, a_{i}\right)+\frac{P}{2} \\
& \leq 2 \sigma P \frac{1}{4 \sigma}+\frac{P}{2}=P .
\end{aligned}
$$


Thus, by induction we have $p\left(a_{i}\right) \leq P$ for all $1 \leq 1 \leq|I|$.

Now, we show that for any $i$ and $k$ with $\min \{i, k\}>1$,

$$
\sum_{j=1}^{\min \{i, k\}-1}\left(\frac{\ell\left(a_{j}\right) \ell\left(a_{k}, a_{i}\right)}{\ell\left(a_{j}, a_{i}\right) \ell\left(a_{k}, a_{j}\right)}\right)^{\kappa} \leq\left(2+\tau^{1 / \kappa}\right)^{\kappa} \tau
$$

Indeed, consider any $1<j<\min \{i, k\}$. Then,

$$
\left(\frac{\ell\left(a_{j}\right)}{\ell\left(a_{j}, a_{i}\right)}\right)^{\kappa}=c\left(a_{j}, a_{i}\right) \leq \tau
$$

which implies

$$
\ell\left(a_{j}\right) \leq \tau^{1 / \kappa} \ell\left(a_{j}, a_{i}\right)
$$

Similarly,

$$
\ell\left(a_{j}\right) \leq \tau^{1 / \kappa} \ell\left(a_{j}, a_{k}\right) .
$$

Thus,

$$
\begin{aligned}
& \quad \frac{\ell\left(a_{j}\right) \ell\left(a_{k}, a_{i}\right)}{\ell\left(a_{k}, a_{j}\right) \ell\left(a_{j}, a_{i}\right)} \\
& \leq \frac{\ell\left(a_{j}\right)\left(\ell\left(a_{k}, a_{j}\right)+\ell\left(a_{j}\right)+\ell\left(a_{j}, a_{i}\right)\right)}{\ell\left(a_{k}, a_{j}\right) \ell\left(a_{j}, a_{i}\right)} \\
& \leq \frac{\ell\left(a_{j}\right)\left(\ell\left(a_{k}, a_{j}\right)+\frac{\tau^{1 / \kappa}}{2} \ell\left(a_{k}, a_{j}\right)+\frac{\tau^{1 / \kappa}}{2} \ell\left(a_{j}, a_{i}\right)+\ell\left(a_{j}, a_{i}\right)\right)}{\ell\left(a_{k}, a_{j}\right) \ell\left(a_{j}, a_{i}\right)} \\
& =\left(1+\frac{\tau^{1 / \kappa}}{2}\right)\left(\frac{\ell\left(a_{j}\right)}{\ell\left(a_{j}, a_{i}\right)}+\frac{\ell\left(a_{j}\right)}{\ell\left(a_{k}, a_{j}\right)}\right)
\end{aligned}
$$

By the generalized mean inequality,

$$
\begin{aligned}
& \left(\frac{\ell\left(a_{j}\right) \ell\left(a_{k}, a_{i}\right)}{\ell\left(a_{j}, a_{i}\right) \ell\left(a_{k}, a_{j}\right)}\right)^{\kappa} \\
& \leq\left(1+\frac{\tau^{1 / \kappa}}{2}\right)^{\kappa}\left(\frac{\ell\left(a_{j}\right)}{\ell\left(a_{j}, a_{i}\right)}+\frac{\ell\left(a_{j}\right)}{\ell\left(a_{k}, a_{j}\right)}\right)^{\kappa} \\
& \leq\left(1+\frac{\tau^{1 / \kappa}}{2}\right)^{\kappa} 2^{\kappa-1}\left(\left(\frac{\ell\left(a_{j}\right)}{\ell\left(a_{j}, a_{i}\right)}\right)^{\kappa}+\left(\frac{\ell\left(a_{j}\right)}{\ell\left(a_{k}, a_{j}\right)}\right)^{\kappa}\right) \\
& =\frac{1}{2}\left(2+\tau^{1 / \kappa}\right)^{\kappa}\left(c\left(a_{j}, a_{i}\right)+c\left(a_{k}, a_{j}\right)\right)
\end{aligned}
$$


Therefore,

$$
\begin{aligned}
& \sum_{j=1}^{\min \{i, k\}-1}\left(\frac{\ell\left(a_{j}\right) \ell\left(a_{k}, a_{i}\right)}{\ell\left(a_{j}, a_{i}\right) \ell\left(a_{k}, a_{j}\right)}\right)^{\kappa} \\
\leq & \frac{1}{2}\left(2+\tau^{1 / \kappa}\right)^{\kappa}\left(\sum_{j=1}^{\min \{i, k\}-1} c\left(a_{j}, a_{i}\right)+\sum_{j=1}^{\min \{i, k\}-1} c\left(a_{k}, a_{j}\right)\right) \\
\leq & \left(2+\tau^{1 / \kappa}\right)^{\kappa} \tau .
\end{aligned}
$$

So, our claim holds.

Next, we show that for any $1 \leq i \leq|I|$,

$$
\sum_{j=1}^{i-1} \frac{p\left(a_{j}\right)}{\ell\left(a_{j}, a_{i}\right)^{\kappa}}<\frac{1}{2 \sigma} \frac{p\left(a_{i}\right)}{\ell\left(a_{i}\right)^{\kappa}} .
$$

In other words, for each link $a_{j}$ the total interference from all preceding links is less than $\frac{1}{2 \sigma}$ times its wanted signal strength. The above inequality holds trivially if $i=1$. So, we assume that $i>1$. Then,

$$
\begin{aligned}
& \sum_{j=1}^{i-1} \frac{p\left(a_{j}\right)}{\ell\left(a_{j}, a_{i}\right)^{\kappa}} \\
& =\sum_{j=1}^{i-1} \frac{2 \sigma \sum_{k=j+1}^{|I|} p\left(a_{k}\right)\left(\frac{\ell\left(a_{j}\right)}{\ell\left(a_{k}, a_{j}\right)}\right)^{\kappa}+2 \frac{\sigma \xi}{\eta} \ell\left(a_{j}\right)^{\kappa}}{\ell\left(a_{j}, a_{i}\right)^{\kappa}} \\
& =2 \sigma \sum_{j=1}^{i-1} \sum_{k=j+1}^{|I|} p\left(a_{k}\right)\left(\frac{\ell\left(a_{j}\right)}{\ell\left(a_{k}, a_{j}\right) \ell\left(a_{j}, a_{i}\right)}\right)^{\kappa}+2 \frac{\sigma \xi}{\eta} \sum_{j=1}^{i-1}\left(\frac{\ell\left(a_{j}\right)}{\ell\left(a_{j}, a_{i}\right)}\right)^{\kappa} \\
& \leq 2 \sigma \sum_{k=2}^{|I|} \sum_{j=1}^{\min \{i, k\}-1} p\left(a_{k}\right)\left(\frac{\ell\left(a_{j}\right)}{\ell\left(a_{k}, a_{j}\right) \ell\left(a_{j}, a_{i}\right)}\right)^{\kappa}+2 \sigma \tau \frac{\xi}{\eta} \\
& =2 \sigma \sum_{k=2}^{|I|} \frac{p\left(a_{k}\right)}{\ell\left(a_{k}, a_{i}\right)^{\kappa}} \sum_{j=1}^{\min \{i, k\}-1}\left(\frac{\ell\left(a_{j}\right) \ell\left(a_{k}, a_{i}\right)}{\ell\left(a_{k}, a_{j}\right) \ell\left(a_{j}, a_{i}\right)}\right)^{\kappa}+2 \sigma \tau \frac{\xi}{\eta} \\
& \leq 2 \sigma \tau\left(2+\tau^{1 / \kappa}\right)^{\kappa} \sum_{k=2}^{|I|} \frac{p\left(a_{k}\right)}{\ell\left(a_{k}, a_{i}\right)^{\kappa}}+2 \sigma \tau \frac{\xi}{\eta} \\
& =\frac{1}{2(1+\sigma)} \sum_{k=2}^{|I|} \frac{p\left(a_{k}\right)}{\ell\left(a_{k}, a_{i}\right)^{\kappa}}+\frac{\left(2+\tau^{1 / \kappa}\right)^{-\kappa}}{2(1+\sigma)} \frac{\xi}{\eta} \\
& =\frac{1}{2(1+\sigma)}\left(\sum_{k=2}^{i-1} \frac{p\left(a_{k}\right)}{\ell\left(a_{k}, a_{i}\right)^{\kappa}}+\frac{p\left(a_{i}\right)}{\ell\left(a_{i}\right)^{\kappa}}+\sum_{k=i+1}^{|I|} \frac{p\left(a_{k}\right)}{\ell\left(a_{k}, a_{i}\right)^{\kappa}}\right)+\frac{\left(2+\tau^{1 / \kappa}\right)^{-\kappa}}{2(1+\sigma)} \frac{\xi}{\eta}
\end{aligned}
$$




$$
\begin{aligned}
& <\frac{1}{2(1+\sigma)}\left(\sum_{k=1}^{i-1} \frac{p\left(a_{k}\right)}{\ell\left(a_{k}, a_{i}\right)^{\kappa}}+\frac{p\left(a_{i}\right)}{\ell\left(a_{i}\right)^{\kappa}}+\frac{1}{2 \sigma} \frac{p\left(a_{i}\right)}{\ell\left(a_{i}\right)^{\kappa}}-\frac{\xi}{\eta}\right)+\frac{\left(2+\tau^{1 / \kappa}\right)^{-\kappa}}{2(1+\sigma)} \frac{\xi}{\eta} \\
& =\frac{1}{2(1+\sigma)} \sum_{k=1}^{i-1} \frac{p\left(a_{k}\right)}{\ell\left(a_{k}, a_{i}\right)^{\kappa}}+\frac{1+\frac{1}{2 \sigma}}{2(1+\sigma)} \frac{p\left(a_{i}\right)}{\ell\left(a_{i}\right)^{\kappa}}-\frac{1-\left(2+\tau^{1 / \kappa}\right)^{-\kappa}}{2(1+\sigma)} \frac{\xi}{\eta} \\
& <\frac{1}{2(1+\sigma)} \sum_{k=1}^{i-1} \frac{p\left(a_{k}\right)}{\ell\left(a_{k}, a_{i}\right)^{\kappa}}+\frac{1+\frac{1}{2 \sigma}}{2(1+\sigma)} \frac{p\left(a_{i}\right)}{\ell\left(a_{i}\right)^{\kappa}} .
\end{aligned}
$$

Therefore,

$$
\sum_{k=1}^{i-1} \frac{p\left(a_{k}\right)}{\ell\left(a_{k}, a_{i}\right)^{\kappa}}<\frac{1+\frac{1}{2 \sigma}}{1+2 \sigma} \frac{p\left(a_{i}\right)}{\ell\left(a_{i}\right)^{\kappa}}=\frac{1}{2 \sigma} \frac{p\left(a_{i}\right)}{\ell\left(a_{i}\right)^{\kappa}} .
$$

Finally, we claim that for any $1 \leq i \leq|I|$, the SINR of $a_{i}$ exceeds $\sigma$. Indeed, for any $1 \leq i \leq|I|$, the SINR of $a_{i}$ is

$$
\begin{aligned}
& \frac{\frac{p\left(a_{i}\right)}{\ell\left(a_{i}\right)^{\kappa}}}{\sum_{j=1}^{i-1} \frac{p\left(a_{j}\right)}{\ell\left(a_{j}, a_{i}\right)^{\kappa}}+\sum_{j=i+1}^{|I|} \frac{p\left(a_{j}\right)}{\ell\left(a_{j}, a_{i}\right)^{\kappa}}+\frac{\xi}{\eta}} \\
& >\frac{\frac{p\left(a_{i}\right)}{\ell\left(a_{i}\right)^{\kappa}}}{\frac{1}{2 \sigma} \frac{p\left(a_{i}\right)}{\ell\left(a_{i}\right)^{\kappa}}+\frac{1}{2 \sigma} \frac{p\left(a_{i}\right)}{\ell\left(a_{i}\right)^{\kappa}}}=\sigma .
\end{aligned}
$$

This completes the proof of Theorem.

Motivated by Theorem [3, we propose an algorithm ShortIS for MISL with power control restricted to short links described as follows. Let $B$ be a subset of short links. A set $S$ of links in $B$ is said to be fit if

$$
\begin{aligned}
& \sum_{b \in S_{\prec}(a)} c(a, b) \leq \tau, \\
& \sum_{b \in S_{\prec}(a)} c(b, a) \leq \tau .
\end{aligned}
$$

The algorithm ShortIS selects a set $I$ of $B$ in two phases.

- Phase 1: Select a fit subset $S$ of $B$ in the ordering $\prec$ in the first-fit manner.

- Phase 2: Let $I$ be the set of links $b$ in $S$ satisfying that

$$
\sum_{a \in S_{\succ}(b)} c(a, b) \leq \frac{1}{4 \sigma} .
$$

Theorem 3 implies that $I$ is independent under the canonical iterative power assignment. We further bound $|I|$ in the next theorem.

Theorem 4. $|I| \geq \alpha(B) / \mu_{2}$, where

$$
\mu_{2}=\frac{2^{\kappa+2}\left(10+\frac{2 \gamma}{\tau}\right)}{\sigma(1-4 \sigma \tau)} .
$$


Proof. We begin with the establishment of the following relation between $|I|$ and $|S|:$

$$
|I|>(1-4 \sigma \tau)|S|
$$

Since

$$
\begin{aligned}
\frac{1}{4 \sigma}|S \backslash I| & <\sum_{b \in S \backslash I} \sum_{a \in S_{\succ}(b)} c(a, b) \leq \sum_{b \in S} \sum_{a \in S_{\succ}(b)} c(a, b) \\
& =\sum_{a \in S} \sum_{b \in S_{\prec}(a)} c(a, b) \leq|S| \tau .
\end{aligned}
$$

we have,

$$
|S \backslash I|<4 \sigma \tau|S|
$$

Therefore,

$$
|I|>(1-4 \sigma \tau)|S|
$$

Let $I^{*}$ be a maximum independent set of $B$ and $p$ be its associated power assignment. Then, $\left|I^{*}\right|=\alpha(B)$. Denote $\phi=2^{-\kappa} \sigma$. By Theorem 1, $I^{*}$ contains a subset $O$ satisfying that $|O| \geq \frac{\phi}{4}\left|I^{*}\right|$ and each link has total relative interference at most $\phi$. In order to prove that $|I| \geq \alpha(B) / \mu_{2}$, it is sufficient to show that

$$
|O| \leq\left(10+\frac{2 \gamma}{\tau}\right)|S| .
$$

To prove this inequality, we partition $O$ as follows. For any link $a \in A$, let $O_{\succ}^{\prime}(a)$ be the set of links $b$ in $O_{\succ}(a)$ satisfying that either the sender of $b$ has distance at most $\ell(a)$ from the receiver of $a$ or the receiver of $b$ has distance at most $\ell(a)$ from the sender of $a$. Let

$$
\begin{aligned}
O^{\prime} & =\bigcup_{a \in S} O^{\prime}(a), \\
O^{\prime \prime} & =O \backslash O^{\prime \prime} .
\end{aligned}
$$

Then, $O \cap S \subseteq O^{\prime}$ and hence $O^{\prime \prime} \subseteq O \backslash S$. We will prove that $\left|O^{\prime}\right| \leq 10|S|$ and $\left|O^{\prime \prime}\right| \leq \frac{2 \gamma}{\tau}|S|$, from which the above inequality holds.

We first claim that for any pair of links $a$ and $b$ in $O$, their senders have distance greater than $\min \{\ell(a), \ell(b)\}$. We prove the claim by contradiction. Assume by contradiction that the distance $t$ between the two senders of some pair of links $a$ and $b$ in $O$ is at most $\min \{\ell(a), \ell(b)\}$. Then,

$$
\begin{aligned}
4 \ell(a) \ell(b) & <\ell(a, b) \ell(b, a) \leq(\ell(a)+t)(\ell(b)+t) \\
& \leq 2 \ell(a) \cdot 2 \ell(b)=4 \ell(a) \ell(b),
\end{aligned}
$$

which is a contradiction. Therefore, our claim holds. Similarly, we can show that for any pair of links $a$ and $b$ in $O$, their receivers have distance greater than $\min \{\ell(a), \ell(b)\}$. 
Next, we show that $\left|O^{\prime}\right| \leq 10|S|$. Using the fact any unit disk can pack at most 5 points of mutual distances greater than one and the claims in the previous paragraph, we have $\left|O_{\succ}^{\prime}(a)\right| \leq 5+5=10$ for any $a \in S$. Therefore,

$$
\left|O^{\prime}\right| \leq \sum_{a \in S}\left|O^{\prime}(a)\right| \leq 10|S|
$$

Finally, we show that $\left|O^{\prime \prime}\right| \leq \frac{2 \gamma}{\tau}|S|$. By Lemma 1, for any $a \in S$,

$$
\begin{aligned}
& \sum_{b \in O_{\succ}^{\prime \prime}(a)} c(a, b)<\gamma, \\
& \sum_{b \in O_{\succ}^{\prime \prime}(a)} c(b, a)<\gamma .
\end{aligned}
$$

Therefore,

$$
\begin{aligned}
2 \gamma|S| & \geq \sum_{a \in S} \sum_{b \in O_{\succ}^{\prime \prime}(a)}[c(a, b)+c(b, a)] \\
& =\sum_{b \in O^{\prime \prime}} \sum_{a \in S_{\prec}(b)}[c(a, b)+c(b, a)] \\
& \geq \sum_{b \in O^{\prime \prime}} \max \left\{\sum_{a \in S_{\prec}(b)} c(a, b), \sum_{a \in S_{\prec}(b)} c(b, a)\right\} \\
& >\tau\left|O^{\prime \prime}\right|
\end{aligned}
$$

which implies

$$
\left|O^{\prime \prime}\right| \leq \frac{2 \gamma}{\tau}|S|
$$

This complete the proof.

\subsection{Approximation Algorithm}

Now, we are ready to describe our approximation algorithm for MISL with power control. Suppose that $B$ is a set of links in $A$. Let $B_{1}$ (respectively, $B_{2}$ ) denote the set of long (respectively, short) links in $B$. Our algorithm proceeds in three steps:

- Step 1: Apply the algorithm LongIS to $B_{1}$ and to select an independent set $I_{1}$ of $B_{1}$ and let $q_{1}$ be the canonical uniform power assignment to $I_{1}$.

- Step 2: Choose $I_{2}$ by the algorithm ShortIS, and let $q_{2}$ be the canonical iterative power assignment to $I_{2}$.

- Step 3: Let $I$ be the larger one between $I_{1}$ and $I_{2}$. If $I=I_{1}$, then output $I$ and $q_{1}$; otherwise, output $I$ and $q_{2}$.

The next theorem establishes the sub-optimality of the output $I$, which implies an approximation bound $16 \mu_{1}+\mu_{2}$ of the above algorithm. 
Theorem 5. $\alpha(B) \leq\left(16 \mu_{1}+\mu_{2}\right)|I|$.

Proof. Let $I^{*}$ be a maximum independent set of $B$ and $p$ be its associated power assignment. By Theorem 2 .

$$
\left|I^{*} \cap B_{1}\right| \leq 16 \mu_{1}\left|I_{1}\right|
$$

On the other hand, by Theorem 4 ,

$$
\left|I^{*} \cap B_{2}\right| \leq \mu_{2}\left|I_{2}\right|
$$

Hence,

$$
\begin{aligned}
\left|I^{*}\right| & =\left|I^{*} \cap B_{1}\right|+\left|I^{*} \cap B_{2}\right| \leq 16 \mu_{1}\left|I_{1}\right|+\mu_{2}\left|I_{2}\right| \\
& \leq\left(16 \mu_{1}+\mu_{2}\right) \max \left\{\left|I_{1}\right|,\left|I_{2}\right|\right\}=\left(16 \mu_{1}+\mu_{2}\right)|I| .
\end{aligned}
$$

So, the the theorem follows.

\section{Discussions}

In this paper, we developed a constant-approximation algorithm for MISL with power control in which all nodes have bounded maximum transmission power and $\kappa>2$. Our algorithm can be generalized to an arbitrary metric. It still achieves a constant approximation bound in the fading metric and a logarithmic approximation bound in the general metric by using the bound on $\gamma$ derived in [8].

The other link scheduling problems including Shortest Link Schedule (SLS), Shortest Fractional Link Schedule (SFLS), Maximum Weighted Independent Set of Links (MWISL), Maximum Multiflow (MMF), and Maximum Concurrent Multiflow (MCMF) have polynomial algorithmic reductions to MISL at the expense of increasing the approximation ratio by a factor $O(\ln \alpha)$ [911]. Our result in this paper implies $O(\ln \alpha)$-approximation algorithms for all these problems.

\section{References}

1. Andrews, M., Dinitz, M.: Maximizing Capacity in Arbitrary Wireless Networks in the SINR Model: Complexity and Game Theory. In: IEEE INFOCOM (2009)

2. Fanghänel, A., Keßelheim, T., Vöcking, B.: Improved algorithms for latency minimization in wireless networks. In: Albers, S., Marchetti-Spaccamela, A., Matias, Y., Nikoletseas, S., Thomas, W. (eds.) ICALP 2009. LNCS, vol. 5556, pp. 447-458. Springer, Heidelberg (2009)

3. Goussevskaia, O., Oswald, Y.A., Wattenhofer, R.: Complexity in geometric SINR. In: Proc. of the 8th ACM MOBIHOC, pp. 100-109 (September 2007)

4. Goussevskaia, O., Halldórsson, M.M., Wattenhofer, R., Welzl, E.: Capacity of Arbitrary Wireless Networks. In: IEEE INFOCOM (2009) 
5. Halldórsson, M.M.: Wireless Scheduling with Power Control. In: Fiat, A., Sanders, P. (eds.) ESA 2009. LNCS, vol. 5757, pp. 361-372. Springer, Heidelberg (2009)

6. Halldórsson, M.M., Mitra, P.: Wireless capacity with oblivious power in general metrics. In: Proceedings of SIAM SODA (2011)

7. Halldórsson, M.M., Wattenhofer, R.: Wireless Communication is in APX. In: ICALP (July 2009)

8. Kesselheim, T.: A Constant-Factor Approximation for Wireless Capacity Maximization with Power Control in the SINR Model. In: Proceedings of SIAM SODA 2011 (2011)

9. Wan, P.-J., Frieder, O., Jia, X., Yao, F., Xu, X.-H., Tang, S.-J.: Wireless Link Scheduling under Physical Interference Model. In: IEEE INFOCOM 2011 (2011)

10. Wan, P.-J., Jia, X., Yao, F.: Maximum independent set of links under physical interference model. In: Liu, B., Bestavros, A., Du, D.-Z., Wang, J. (eds.) WASA 2009. LNCS, vol. 5682, pp. 169-178. Springer, Heidelberg (2009)

11. Wan, P.-J., Xu, X.-H., Frieder, O.: Shortest Link Scheduling with Power Control under Physical Interference Model. In: Proceedings of the 6th International Conference on Mobile Ad-hoc and Sensor Networks, MSN 2010 (2010)

12. Xu, X.-H., Tang, S.-J.: A Constant Approximation Algorithm for Link Scheduling in Arbitrary Networks under Physical Interference Model. In: The Second ACM International Workshop on Foundations of Wireless Ad Hoc and Sensor Networking and Computing (May 2009) 